\title{
A VARIATIONAL PROBLEM MODELLING BEHAVIOR OF UNORTHODOX SILICON CRYSTALS *
}

\author{
J. HANNON ${ }^{1}$, M. MARCUS ${ }^{2}$ AND ViCTOR J. MizEL ${ }^{3}$
}

\begin{abstract}
Controlling growth at crystalline surfaces requires a detailed and quantitative understanding of the thermodynamic and kinetic parameters governing mass transport. Many of these parameters can be determined by analyzing the isothermal wandering of steps at a vicinal ["step-terrace"] type surface [for a recent review see [4]]. In the case of orthodox crystals one finds that these meanderings develop larger amplitudes as the equilibrium temperature is raised (as is consistent with the statistical mechanical view of the meanderings as arising from atomic interchanges). The classical theory due to Herring, Mullins and others [5], coupled with advances in real-time experimental microscopy techniques, has proven very successful in the applied development of such crystalline materials. However in 1997 a series of experimental observations on vicinal defects of heavily boron-doped Silicon crystals revealed that these crystals were quite unorthodox in the sense that a lowering of the equilibrium temperature led to increased amplitude for the isothermal wanderings of a step edge [3]. In addition, at low temperatures the step profile adopted a periodic saw-tooth structure rather than the straight profile predicted by the classical theories. This article examines a stored free energy model for such crystals involving a (higher order) Landau/de Gennes type "order parameter" term and provides a proof for the existence of a minimizer.
\end{abstract}

Mathematics Subject Classification. 49J45, 49S05.

Received May 31, 2002.

\section{The Classical MODEL}

The "classical" model for the free energy of certain crystals is given by

$$
J_{1}(y)=\int_{0}^{S} \beta(\theta) \mathrm{d} s
$$

where $s$ is arclength and $y$ is a function defined on a fixed interval $[0, L]$ whose graph is the locus under consideration:

$$
y \in W^{1,1}(0, L), \quad \theta=\arctan y^{\prime} \in[-\pi / 2, \pi / 2]
$$

Keywords and phrases: Landau/de Gennes order parameter, parametric problem.

* Dedicated to the Memory of Bill Mullins.

1 IBM, Yorktown Heights, NY 10598, U.S.A.

2 Technion, Haifa 32000, Israel

3 Carnegie Mellon University, Pittsburgh, PA 15213, U.S.A.; e-mail: vm09@andrew.cmu.edu 
while $\beta$ is a positive $\pi$-periodic function which satisfies $\beta(\theta)=\beta(-\theta)$. An additional very important property of $\beta$ is

$$
\max \beta=\beta(0), \min \beta=\beta(\pi / 2) .
$$

Minimization of $J_{1}$ subject to appropriate boundary data is clearly a parametric variational problem [so that the graph could in general possess vertical sections]. It is closely related to the variational problem defining the Wulff crystal shape as that shape for a domain of prescribed area such that the boundary integral with respect to arclength involving the integrand in $J_{1}$ [referred to as the surface tension] attains its minimum value. Since the existence and convexity, as well as uniqueness up to translation, of such a figure [also referred to as the $\beta$ crystal] has been demonstrated ( $c f$. for example $[1,2]$ ), it readily follows that for a given $L$ any locus lying fully on one side of the given horizontal segment of length $L$ on which $J_{1}$ attains its minimum value would have to be a section of the boundary of (some dilation of) the $\beta$ crystal. In fact, supposing the area below this minimizing locus to be $A_{0}$, there is a dilation $\mathrm{C}$ of the $\beta$ crystal for which a horizontal chord of length $\mathrm{L}$ cuts off an area $A_{0}$. The boundary section of $\mathrm{C}$ lying above this region is therefore the minimizer in question. In particular, the locus in question would be a convex curve. Consequently, a nonconvex minimizing locus cannot occur unless it lies partly above and partly below the given segment, in which case the sections lying above the segment and the sections lying below it would necessarily consist of unions of convex figures. A necessary condition which any minimizing locus must satisfy is associated with the quantity labeled stiffness which is defined by $B(\theta)=\beta^{\prime \prime}(\theta)+\beta(\theta)$. We proceed to discuss the structure of such minimizers in the simpler case in which $B(0)<0$, which is the one relevant to the experiments in [3]. For this case we will show in particular that each minimizing locus is indeed the graph of a function $y$ of the horizontal $x$-coordinate. Put

$$
\beta^{*}(z)=\beta(\arctan z), z \in[-\infty, \infty], \quad \arctan z \in[-\pi / 2, \pi / 2] .
$$

Then $\beta^{*}$ is a positive function which has its maximum at zero and its minimum at $\infty$. We now rewrite $J_{1}$ in the form

$$
J_{1}(y)=\int_{0}^{L} \beta^{*}\left(y^{\prime}\right) \sqrt{1+\left(y^{\prime}\right)^{2}} \mathrm{~d} x .
$$

Proposition 1.1. Suppose that $\beta$ is even and belongs to $C^{2}(\mathbb{R})$. If

$$
B(0)=\beta^{\prime \prime}(0)+\beta(0)<0,
$$

then there exists a positive number $\gamma$ such that every function $y$ satisfying

$$
y \in W_{0}^{1,1}(0, L), \quad\left|y^{\prime}\right|=\gamma \text { a.e. in }(0, L)
$$

is a minimizer of $J_{1}$.

Proof. The function $y$ is clearly a minimizer if

$$
G(z):=\beta^{*}(z) \sqrt{1+z^{2}}=\alpha, \text { a.e. }
$$

where $z=y^{\prime}$, with $\alpha=\min G$.

In that event $\min J_{1}=L \alpha$. Under assumption (5) the function $G$ does not attain its minimum value at zero. Since $G(z) \rightarrow \infty$ as $z \rightarrow \pm \infty, G$ possesses a minimum $\alpha$ which is attained at one or more positive arguments $\gamma$. The set $M_{G}:=\{z>0: G(z)=\alpha\}$ need not be a singleton, so that every function $y \in W_{0}^{1,1}(0, L)$ satisfying $\left|y^{\prime}\right| \in M_{G}$ a.e. is a minimizer of $J_{1}$. 
Now the connection to the Wulff model led us to impose the constraint $y(0)=y(L)=0$ on minimizers. On the other hand, physical arguments lead to a somewhat weaker constraint formulated as follows:

$$
1 / L \int_{0}^{L} \mathbf{n} \sqrt{1+\left(y^{\prime}\right)^{2}} \mathrm{~d} x=(0,1)
$$

where $\mathbf{n}(x)=\left(-y^{\prime}(x), 1\right) / \sqrt{1+\left(y^{\prime}\right)^{2}}$ denotes the outer unit normal to the graph of $y$ at $x$ with $y \in W^{1,1}(0, L)$. This yields

$$
\int_{0}^{L} y^{\prime} \mathrm{d} x=0 \Rightarrow y(0)=y(L)
$$

\section{THE NEW MODEL}

Since the classical model led (in the $B(0)<0$ case) to a variational problem with an infinite number of solutions, even under the constraint $y(0)=y(L)=0$, most of which do not conform to the experimental data we wish to model, there was need of improvement. One suggestion was to add a curvature term to the free energy,

$$
J_{2}(y)=\int_{0}^{L}\left(\beta^{*}\left(y^{\prime}\right)+\rho \frac{\left(y^{\prime \prime}\right)^{2}}{\left(1+\left(y^{\prime}\right)^{2}\right)^{3}}\right) \sqrt{1+\left(y^{\prime}\right)^{2}} \mathrm{~d} x
$$

where $\rho$ is a positive scalar. Here we have taken the square of the curvature; one could of course try other powers. The main point is that there is a cost exacted for changing direction, so that this should not occur too frequently. However it is then clear that this model will select among the possible minimizers associated with the classical model, a minimizer which has the fewest changes of direction, e.g. with $\gamma \in M_{G}$ that solution $y$ such that $y^{\prime} \equiv \gamma$ on $[0, L / 2)$ and $y^{\prime} \equiv-\gamma$ on $(L / 2, L]$. Of course the solution would be slightly modified, with a rounded corner at $L / 2$. The concern is that such a solution likewise fails to correspond to the experimental data we wish to model. Therefore a further modification of the model is needed. It became clear that adding a term which exacts a cost in elastic energy for vertical displacement may achieve our aim, and such a term is consistent with physical reasoning. Therefore we propose to study the following model,

$$
J(y)=\int_{0}^{L}\left(\beta^{*}\left(y^{\prime}\right)+\rho \frac{\left(y^{\prime \prime}\right)^{2}}{\left(1+\left(y^{\prime}\right)^{2}\right)^{3}}+\sigma y^{2}\right) \sqrt{1+\left(y^{\prime}\right)^{2}} \mathrm{~d} x
$$

where $\sigma$ and $\rho$ are positive scalars. For the sake of simplicity we assume for the present discussion that $\alpha=\min G$ occurs at precisely one point $\gamma>0$. It is then plausible that the new model will select from among the minimizers of $J_{1}$ a solution $y$ such that for some integer $k, y^{\prime} \equiv \gamma$ in the intervals $((2 j-1) L / 2 k,(2 j+1) L / 2 k)$ for $j \leq 2 k-1$ even, $y^{\prime} \equiv-\gamma$ in the intervals $((2 j-1) L / 2 k,(2 j+1) L / 2 k)$ for $j$ odd, and $y^{\prime} \equiv \gamma$ on $(0, L / 2 k)$ and $((2 k-1) L / 2 k, L)$. Of course the actual solution would be a perturbation of the above with corners rounded off. The number $k$ of triangles would be determined so as to minimize the net sum consisting of $k$ costs for changes in curvature compensated by the lowered cost resulting from the decreased displacements from the axis [compare with our earlier discussion concerning the minimizer for $\left.J_{2}\right]$. Note that if one considers another intermediary model $J_{3}$ which is the form of $J$ when $\rho=0$, then there is no minimizer since increasing the number of triangles and hence lowering their heights decreases the energy. That is inf $J_{3}=\min J_{1}$, but the infimum is not attained. 


\section{The existence theorem}

In view of the above discussion we are led to the following variational problem, where $L, \rho$, and $\sigma$ are prescribed positive scalars,

$$
\begin{aligned}
& J(\theta)=\int_{0}^{S}\left[\rho\left(\theta^{\prime}\right)^{2}+\beta(\theta)+\sigma \mathrm{y}^{2}\right] \mathrm{d} s \rightarrow \inf \\
& y(s)=y(0)+\int_{0}^{s} \sin \theta(\tau) \mathrm{d} \tau
\end{aligned}
$$

where $\theta \in W^{1,2}(0, S)$ is subject to the following constraints:

$$
\theta(s) \in[-\pi / 2, \pi / 2], \int_{0}^{S} \cos \theta(\tau) \mathrm{d} \tau=L, \int_{0}^{S} \sin \theta(\tau) \mathrm{d} \tau=0 .
$$

The first constraint expresses the condition that the curve $(x, y)=(x(s), y(s))$ does not "reverse", the second is the condition that the $x$-interval is $[0, L]$, while the third is the condition $y(0)=y(S)$. Note that $y_{0}=y(0)$ and $S$ are not prescribed so that the infimum is taken over all $S \geq L, \theta \in W^{1,2}(0, S)$, and all real $y_{0}$. However it is easy to see that the for any given function $\theta \in W^{1,2}(0, S)$ the optimal choice of $y(0)$ is that for which the function $y$ has mean zero, i.e.

$$
y(0)=1 / S \int_{0}^{S} s \sin \theta(s) \mathrm{d} s .
$$

Therefore, assuming that $y(0)$ is given by $(13)$, the infimum in problems $(11,12)$ is taken over

$$
Z:=\left\{(S, \theta): S \geq L, \theta \in W^{1,2}(0, S)\right\}
$$

Theorem 3.1. Problems (11, 12) possesses a solution.

Remark 3.1. Note that the theorem does not exclude the possibility that a minimizer $(S, \theta)$ satisfies $|\theta|=\pi / 2$ on one or more nondegenerate intervals. If this occurs then the locus of the curve $s \mapsto(x(s), y(s)), s \in(0, S)$ is not the graph of a function defined on $[0, L]$.

Proof. Let $\left\{\left(S_{n}, \theta_{n}\right)\right\}$ be a minimizing sequence. Since, $J\left(\theta_{n}\right) \geq S_{n} \inf \beta$ and $\inf \beta>0$ it follows that $\left\{S_{n}\right\}$ is bounded. Therefore, in view of (13), $\left\{y_{n}(0)\right\}$ is bounded. Consequently, taking a subsequence if necessary, we may assume that

$$
S_{n} \rightarrow S^{*}, \quad y_{n}(0) \rightarrow y_{0}^{*} .
$$

In addition $\left\{\left\|\theta_{n}^{\prime}\right\|_{L_{2}\left(0, S_{n}\right)}\right\}$ is bounded. Since each function $\theta_{n}$ assumes values in $[(-\pi, \pi]$, it follows that $\left\{\left\|\theta_{n}\right\|_{W^{1,2}\left(0, S_{n}\right)}\right\}$ is bounded.

If $S_{n} \geq S^{*}$ put $\theta_{n}^{*}=\left.\theta_{n}\right|_{\left[0, S^{*}\right]}$. If $S_{n}<S^{*}$ define the function $\theta_{n}^{*}$ in $\left[0, S^{*}\right]$ as follows

$$
\theta_{n}^{*}(s)= \begin{cases}\theta_{n}(s) & \text { if } 0 \leq s \leq S_{n} \\ \theta_{n}\left(S_{n}\right) & \text { otherwise }\end{cases}
$$

Then $\left\{\theta_{n}^{*}\right\}$ is bounded in $W^{1,2}\left(0, S^{*}\right)$. By extracting a subsequence we may assume that $\left\{\theta_{n}^{*}\right\}$ converges weakly in $W^{1,2}\left(0, S^{*}\right)$ to a function $\theta^{*}$. Hence

$$
\begin{aligned}
\theta_{n}^{*} & \rightarrow \theta^{*} \\
y_{n}^{*}(s):=\int_{0}^{s} \sin \theta_{n}^{*}(\sigma) \mathrm{d} \sigma+y_{n}(0) & \rightarrow y^{*}(s):=\int_{0}^{s} \sin \theta^{*}(\sigma) \mathrm{d} \sigma+y_{0}^{*}
\end{aligned}
$$


uniformly in $\left[0, S^{*}\right]$. Consequently $\theta^{*}$ satisfies the constraints (12) with $S=S^{*}$ and

$$
J\left(\theta^{*}\right) \leq \liminf J\left(\theta_{n}^{*}\right)
$$

If $Y$ is a bound for $\left\{\sup _{\left[0, S_{n}\right]}\left|y_{n}\right|\right\}$ then, bearing in mind that $\beta(\theta) \leq \beta(0)$,

$$
J\left(\theta_{n}^{*}\right) \leq J\left(\theta_{n}\right)+\left(\beta(0)+a Y^{2}\right)\left(S^{*}-S_{n}\right)_{+}
$$

By $(14,17)$, and $(18)$

$$
J\left(\theta^{*}\right) \leq \lim J\left(\theta_{n}\right)=\inf _{Z} J .
$$

Thus $\theta^{*}$ is a minimizer of $J$ subject to (12). This proves the theorem.

One of us (VJM) wishes to express his appreciation to Prof. Art Heinricher of Worcester Polytechnic Institute for his extremely helpful comments concerning the importance of the parametric nature of the variational problem involved here, as well as for his help in locating some of the control theory literature developed for dealing with such problems in great generality. Of course, as seen above, our present results turned out to be achievable by a comparatively elementary direct argument not requiring the use of the more sophisticated apparatus available in the control literature. In addition both MM and VJM owe a profound debt to Prof. Ari Leizarowitz of the Technion for his many important comments on this variational problem; in particular, his efforts to determine whether the minimizers are necessarily graphs were extremely enlightening for both of us. Finally, one of us (VJM) wishes to express his appreciation to the US National Science Foundation for its partial support of this research under Grant 0072816 as well as to the Technion for its support of a visit during which much of this research was carried out.

\section{REFERENCES}

[1] B. Dacorogna and C.E. Pfister, Wulff theorem and best constant in Sobolev inequality. J. Math. Pures Appl. 71 (1992) 97-118.

[2] I. Fonseca, The Wulff theorem revisited. Proc. Roy. Soc. Lond. A 432 (1991) 125-145.

[3] J. Hannon et al., Step Faceting at the (001) Surface of Boron Doped Silicon. Phys. Rev. Lett. 79 (1997) $4226-4229$.

[4] H.C. Jeong and E.D. Williams, Steps on Surfaces: Experiment and Theory. Surface Sci. Reports 34 (1999) $175-294$.

[5] W.W. Mullins, Theory of thermal grooving. J. Appl. Phys. 28 (1957) 333-339.

[6] J.E. Taylor, II-Mean curvature and weighted mean curvature. Acta Metall. Mater. 40 (1992) 1475-1485. 